\title{
INTERSTELLAR SCINTILLATIONS AND NEUTRON STAR KINEMATICS
}

\author{
J.M. Cordes \\ Astronomy Department \\ Cornell University \\ Ithaca, NY 14853 \\ U.S.A.
}

\begin{abstract}
The interstellar scintillation technique for measuring neutron star speeds is described and results are given for 71 radio pulsars. The mean transverse neutron star speed is $100 \mathrm{~km} \mathrm{~s}^{-1}$ and the distribution extends to $300 \mathrm{~km} \mathrm{~s}^{-1}$. The transverse speed correlates with the $\mathrm{z}$ velocity derived independently using distance from the galactic plane, consistent with most neutron stars having been born near the galactic plane. A correlation of transverse speed with the quantity $\mathrm{P} \dot{\mathrm{P}} \propto$ (magnetic moment) $^{2}$ is a general property of the neutron star population. Monte Carlo simulations of the progenitors of neutron stars show that the velocity distribution is inconsistent with the disruption of binary systems solely by symmetric supernova explosions. Either explosions are asymmetric or there are additional accelerations of neutron stars after their formation.
\end{abstract}

\section{INTRODUCTION}

As with any stellar population, the space velocities of neutron stars are informative about the processes by which the objects are formed. Radio pulsars move (translationally) roughly 10 times faster than their progenitor stars. The mechanisms which may explain the large velocities include the disruption of compact binaries by supernova explosions (Zwicky 1957; Blaauw 1961; Radhakrishnan 1984); asymmetry of supernova explosions (Anderson and Lyne 1983); and asymmetric magnetic dipole radiation (Harrison and Tademaru 1975). At present there is no concensus as to whether one or more of these processes dominates the situation. To make progress, a large sample of velocities is necessary to establish the generality of any derived distribution and to establish correlations with other pulsar parameters. This review discusses such a sample of measurements and its implications.

There are three methods for measuring transverse velocities of radio pulsars. These are (1) direct proper motion measurements using interferometry; (2) pulse timing measurements; and (3) interstellar scintillations, measurement of which yield the motion of a diffraction pattern which, in turn, is related to the neutron star speed. Lyne (this volume) has outlined the first two methods. In this paper I review the scintillation method, describe results obtained on a large sample of radio pulsars, and draw conclusions about mechanisms contributing to the space velocities. 


\section{INTERSTELLAR SCINTILLATIONS}

Figure 1 shows the relevant geometry for scintillations. Pulsars are the most compact sources known in radio astronomy and are emitters of spatially coherent (but temporally incoherent) radiation. Propagation through turbulent plasma in the interstellar medium (ISM) distorts the wavefronts according to refractive index fluctuations described by the electron density and the cold plasma dispersion relation. Birefringence is negligible (Simonetti, Cordes, and Spangler 1984). Under the condition that the phase difference between wavefront components arriving from different angles is more than one radian, a deeply modulated diffraction pattern results, through which the Earth moves. The diffraction pattern is random but has well determined characteristic spatial and frequency scales even though the electron density itself appears to have no well defined spatial scale. In fact, the electron density is consistent with a Kolmogorov (power law) wavenumber spectrum (Armstrong, Cordes, and Rickett 1981; Cordes, Weisberg, and Boriakoff 1985). Diffraction scales are well determined because these are determined by the spatial scale in the medium that produces 1 radian of phase variation.

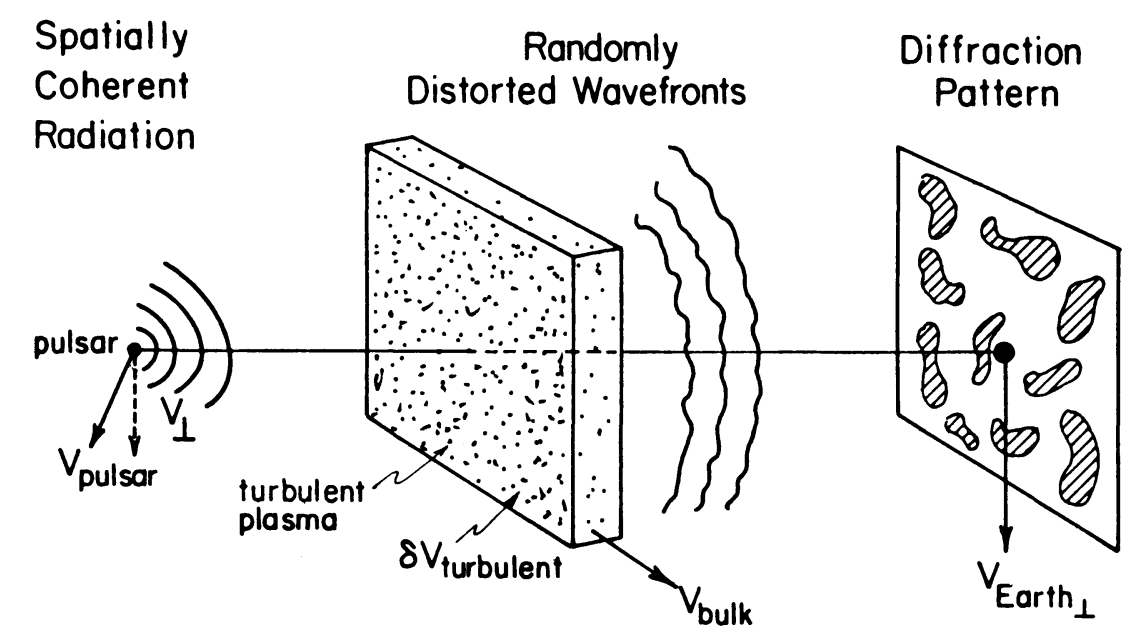

Figure 1 Geometry for scattering of pulsar radiation.

Interstellar scattering is a topic of considerable interest because effects are manifest in many kinds of measurements. These include temporal broadening and time-of-arrival variations of pulsar pulses $\left(10^{-6}\right.$ to $\left.1 \mathrm{sec}\right)$; angular broadening and angle-of-arrival variations of images of pulsars and active galactic nuclei $\left(10^{-6}\right.$ to $\left.1 \mathrm{arc} \mathrm{sec}\right)$; refractive intensity variations on time scales of days to years (Rickett, Coles, and Bourgois 1984); and the diffractive intensity variations that are of interest here and which have time scales of seconds to hours.

\subsection{Observables}

Measurements of intensity as a function of time and frequency ('dynamic spectra') show $\sim 100 \%$ modulations having characteristic time and frequency scales that are related to the spatial scale of the diffraction pattern. Dynamic spectra are 
usually obtained with an autocorrelation spectrometer (or sometimes an analog filter bank) that is gated synchronously with the periodic pulsar signal (for signal to noise enhancement), using a total bandwidth of 0.1 to $10 \mathrm{MHz}$, integration times of $10 \mathrm{sec}$, and total observation times between $20 \mathrm{~min}$ and $2 \mathrm{hr}$. Typical radio frequencies are from 0.3 to $1.4 \mathrm{GHz}$.

Dynamic spectra show fluctuations that are dominated by interstellar scintillations but also include intrinsic pulsar variations; interplanetary and ionospheric scintillations; and any gain variations of the telescope/receiver. All of these additional effects are broadband and therefore produce only additional temporal modulations of the intensity over the spectrometer bandwidth. By using different calibration schemes, we have shown that these additional effects have little influence on the velocities discussed below.

Estimation of the characteristic time and frequency scales is done by calculating the two dimensional intensity autocovariance function (ACV). Letting $\delta I(\nu, t)$ be the deviation of the intensity from the mean, the intensity ACV is $\langle\delta I(\nu, t) \delta I(\nu+\delta \nu, t+\delta t)\rangle$, where angular brackets denote averaging over the receiver bandwith and over time. Figure 2 shows a representative ACV. The widths (HWHM) of the slices along the time and frequency lag axes are the characteristic scintillation scales. These are denoted $\Delta t_{d}$ and $\Delta \nu_{d}$ where the subscript $\mathrm{d}$ implies the quantities are 'diffractive' in origin.
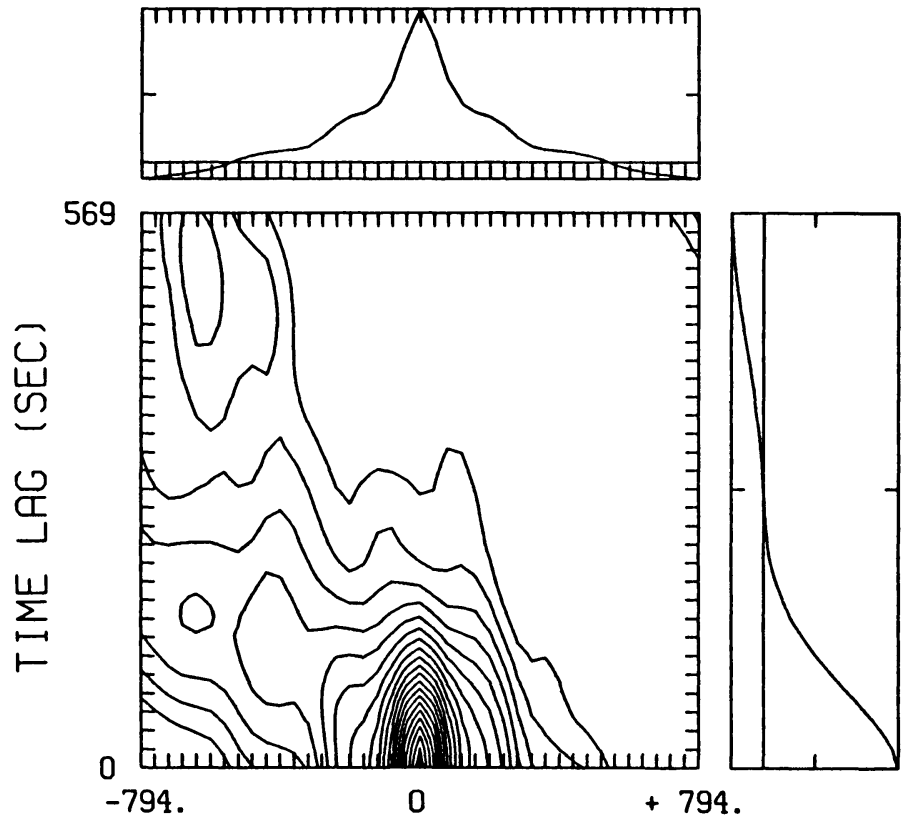

Figure 2 Intensity autocovariance of dynamic spectra with slices along the time lag and frequency lag axes.

\section{FREQUENCY LAG (KHZ)}

\subsection{Theoretical Relations}

Let $\theta_{d}$ be the characteristic diffraction angle and $\mathrm{D}$ be the distance to a pulsar. The diffraction pattern has a spatial scale $\ell_{d} \sim \lambda /\left(2 \pi \theta_{d}\right)$ which is (for radio frequencies of interest) much less than the Fresnel scale $\sqrt{\lambda D} \sim 10^{11} \mathrm{~cm}$. The diffractive 
frequency scale is $\Delta \nu_{d} \sim 2 c /\left(D \theta_{d}{ }^{2}\right) \ll \nu$. Temporal variations are caused by two effects: 1) convection of the diffraction pattern through the line of sight and 2) true time variations that would be seen if all bulk velocities are zero and would be due to turbulent motions of the medium. The velocities that must be considered include:

1. Pulsar velocities

2. Interstellar medium

a. bulk (galactic rotation)

b. random (turbulent)

3. Earth orbital motion

$$
V_{\perp} \quad=0 \rightarrow 300 \mathrm{~km} \mathrm{~s}^{-1}
$$

$$
\begin{aligned}
V_{\text {bulk }} & =0 \rightarrow 150 \mathrm{~km} \mathrm{~s}^{-1} \\
\delta V_{\text {turbulent }} & \leq 10 \mathrm{~km} \mathrm{~s}^{-1} \\
V_{\text {Earth }} & \leq 30 \mathrm{~km} \mathrm{~s}^{-1}
\end{aligned}
$$

Differential galactic rotation is important for interferometric or timing determinations of proper motion (for distant pulsars), but is negligible for scintillation measurements (Cordes 1986; discussed below). Turbulent motions potentially affect all three kinds of measurements but in practice are probably negligible. Evidently, refractive image wandering or temporal wandering is too small to strongly influence interferometric and timing measurements.

For scintillations, we argue that convection is the dominant cause of time variation and that turbulent speeds in the ISM must be small compared to the average neutron star speed. If turbulent motions are negligible, the diffractive time scale is simply $\Delta t_{d} \sim \ell_{d} / V_{\perp}$, where $V_{\perp}$ is an effective velocity. Thus an estimator for this velocity is

$$
V_{\perp} \equiv \frac{\ell_{d}}{\Delta t_{d}} \sim \frac{\sqrt{c D \Delta \nu_{d}}}{2 \pi \nu \Delta t_{d}} \sim 10^{4.1} \mathrm{~km} \mathrm{~s}^{-1}\left(\Delta \nu_{\mathrm{d}} \mathrm{D}_{\mathrm{kpc}}\right)^{1 / 2}\left(\nu_{\mathrm{GHz}} \Delta \mathrm{t}_{\mathrm{d}}\right)^{-1}
$$

where the last approximate equality holds for $\Delta \nu_{d}$ in $\mathrm{MHz}, \Delta t_{d}$ in sec, $\mathrm{D}$ in kpc, and $\nu$ in $\mathrm{GHz}$. In eqn (1), the spatial scale $\ell_{d}$ is estimated using the diffraction bandwidth and the distance. The velocity estimator has the same form independent of the electron density wavenumber spectrum, although the numerical coefficient in front depends on the spectrum and on how scattering material fills the line of sight. Typical numbers are $\theta_{d} \sim 10^{-3}$ arc sec, $\ell_{d} \sim 10^{4} \mathrm{~km}, \Delta \nu_{d} \sim \mathrm{kHz} \rightarrow \mathrm{MHz}$, and $\Delta t_{d} \sim \sec \rightarrow \min$ for $V_{\perp} \sim 100 \mathrm{~km} \mathrm{~s}^{-1}$.

The utility of the velocity estimator in equation (1) is that it is much more efficient to measure the frequency scale using a single antenna than to measure the spatial scale directly using intensity interferometry. Weak pulsars can also be measured using large antennas such as the $305 \mathrm{~m}$ Arecibo telescope.

How good is the velocity estimator? One test involves plotting the quantity $\sqrt{\Delta \nu_{d}} / \nu$ against $\Delta t_{d}$, as shown in Figure 3 for two pulsars. The plotted quantities are linearly related and demonstrate that the spatial scale may indeed be estimated from the diffraction bandwidth. This would not be true if turbulent motions were large. The linear relations therefore imply $\delta V_{\text {turbulent }} \ll$ bulk velocities. An additional test involves plotting $V_{\perp}$ against orbital phase for the binary pulsar PSR 0655+64 (Figure 4). The variation with orbital phase demonstrates that the neutron star speed dominates the motion of the diffraction pattern across the line of sight.

\subsection{Comparison of Scintillations and Interferometry}

Table 1 compares the scintillation and interferometric techniques. In contrast to interferometry, for which the spatial resolution at the source $(\propto D / \nu)$ degrades 


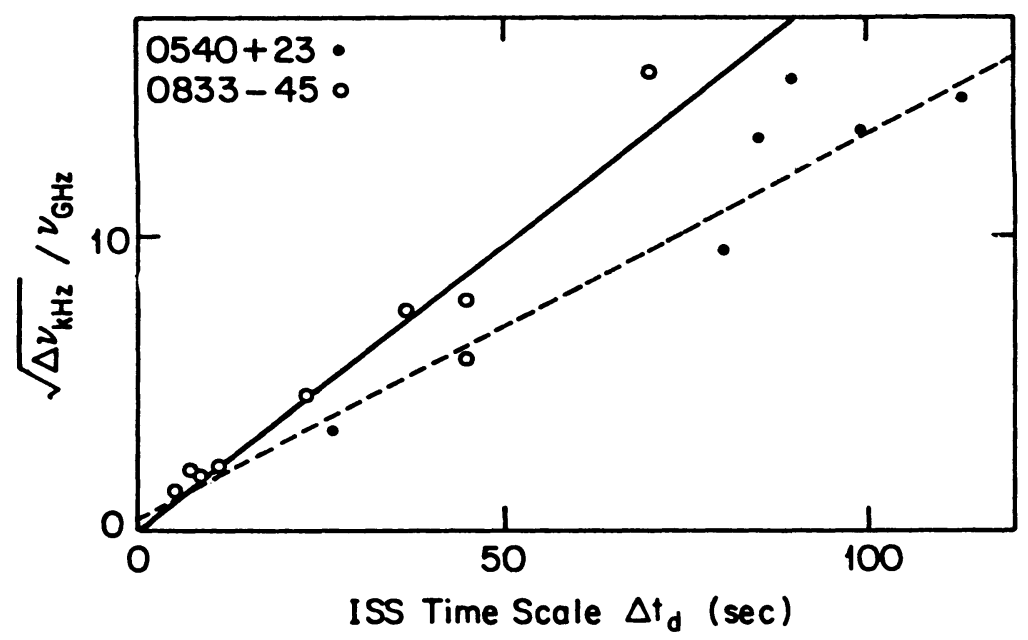

Figure 3 Plot of $\sqrt{\Delta \nu_{d}} / \nu$ vs. $\Delta \mathrm{t}_{\mathrm{d}}$

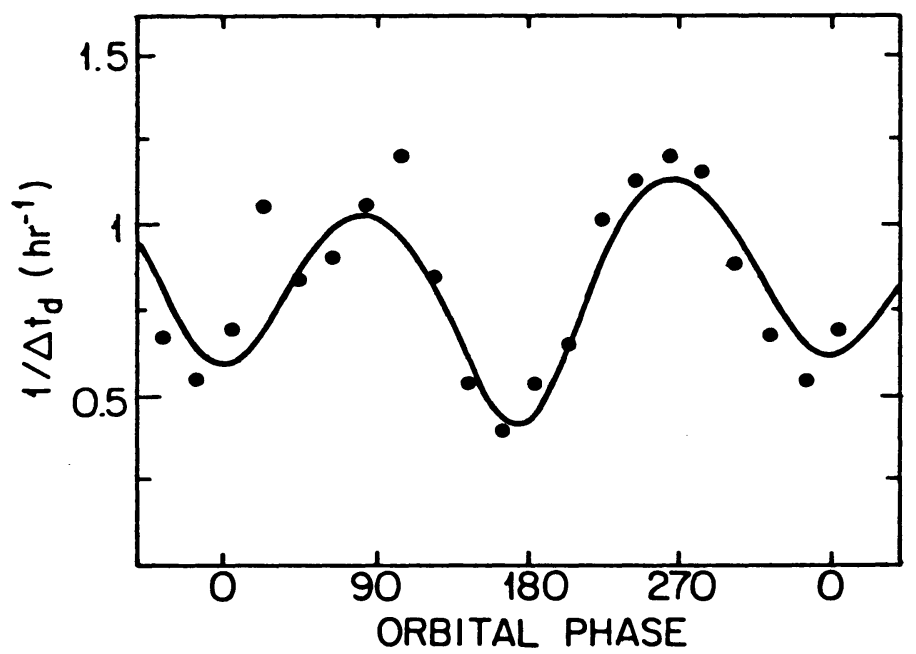

Figure 4 Reciprocal diffraction time scale plotted against orbital phase for the binary pulsar 0655+64 (after Lyne 1984).

for distant pulsars, the scintillation resolution gets better for distant pulsars. For this reason, scintillations are applicable to distant, low-velocity pulsars whereas interferometry is not. Another advantage is that the scintillation velocity depends less strongly on the distance estimate. Most pulsar distances are estimated from the pulsar dispersion measure combined with a model for the interstellar electron density. Such distances, calibrated with a few independently known distances (from HI absorption, parallax, and optical measurements), are thought to have factor of 2 accuracy (e.g. Lyne, Manchester, and Taylor 1985).

Differential galactic rotation contributes to interometry derived proper motions because the interferometer phase is calibrated using extragalactic objects. 
TABLE 1: COMPARISON OF TECHNIQUES

\begin{tabular}{|c|c|c|}
\hline PROPERTY & SCINTILLATIONS & INTERFEROMF \\
\hline $\begin{array}{l}\text { spatial resolution: } \\
\text { (at source) }\end{array}$ & $\begin{array}{l}10^{4} \mathrm{~km} \nu^{6 / 5} D^{-3 / 5} \\
(\nu \text { in } \mathrm{GHz}, \mathrm{D} \text { in } \mathrm{kpc})\end{array}$ & $\begin{array}{l}10^{8} \mathrm{~km}(b \nu)^{-1} D \\
(\mathrm{~b}=1000 \mathrm{~km})\end{array}$ \\
\hline velocity estimates: & $V \propto D^{1 / 2}$ & $V \propto D$ \\
\hline $\begin{array}{l}\text { differential } \\
\text { galactic rotation: }\end{array}$ & insensitive & includes \\
\hline Selection against: & large V, large D & small $\mathrm{V}$, large $\mathrm{D}$ \\
\hline \multirow[t]{3}{*}{$\begin{array}{l}\text { Advantages and } \\
\text { Disadvantages: }\end{array}$} & $\begin{array}{l}1 \text { hour on large } \\
\text { single dish antennas }\end{array}$ & $\geq 1$ year \\
\hline & $\begin{array}{l}\text { scalar velocity } \\
\text { (vector possible) }\end{array}$ & vector velocity \\
\hline & $\begin{array}{l}\text { bias due to scattering } \\
\text { leverage effects }\end{array}$ & \\
\hline
\end{tabular}

With scintillations, however, differential galactic rotation is nearly cancelled out because the scintillation time scale is determined by relative motion of the line of sight and scattering material. Scintillations provide only a scalar velocity and may be biased by leverage effects if enhanced scattering occurs near the pulsar or near the Earth. It is known that distant, low galactic latitude objects are prone to enhanced scattering (Cordes, Weisberg, and Boriakoff 1985). For individual objects, enhanced scattering can be diagnosed by a smaller than expected diffraction bandwidth. It may be feasible to obtain vector velocities with scintillations (Cordes 1984) using multiepoch measurements, depending on the amplitude of refractive effects which also modulate the scintillation parameters on month to year time scales.

\section{NEUTRON STAR VELOCITIES}

Figure 5 shows the distribution of transverse speeds derived from scintillations for 71 objects and the distribution for the 26 objects studied with interferometry (Lyne, Anderson, and Salter 1982; hereafter LAS). The distributions are consistent with each other and show a large mean speed $\sim 100 \mathrm{~km} \mathrm{~s}^{-1}$ and a tail extended to $\sim 400 \mathrm{~km} \mathrm{~s}^{-1}$.

How do selection effects affect the shape of the derived distribution? Probably negligibly. The pulsars in the scintillation and the LAS samples were chosen primarily on the basis of their flux density and declination. Selection effects in the original pulsar searches are generally strong with respect to luminosity and galactic coordinates but these do not produce any strong velocity selection. Distance errors 


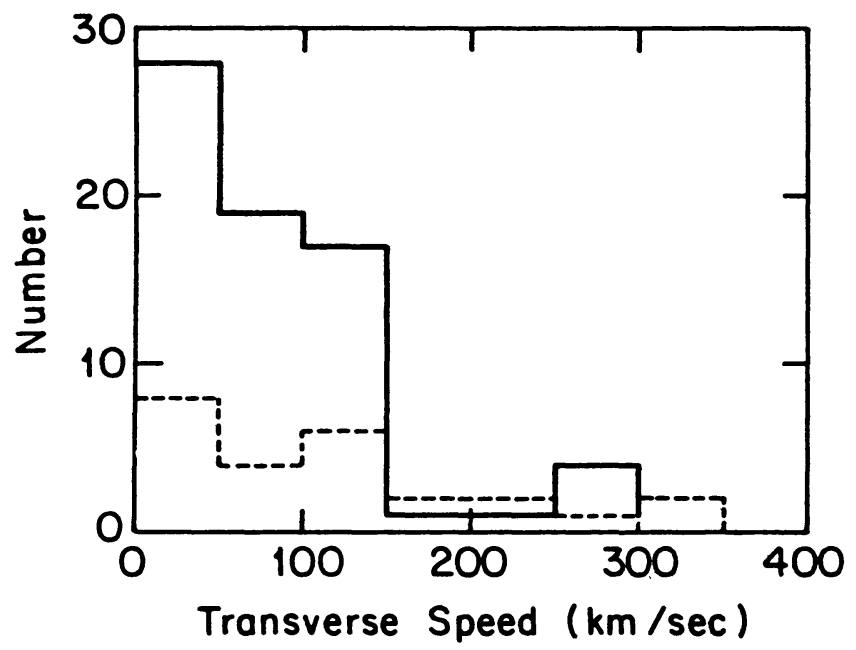

Figure 5 Histogram of transverse neutron star speeds. (Solid line) Scintillation speeds for 71 objects (Dashed line) Interferometric speeds for 26 objects (Lyne, Anderson, and Salter 1982).

cause the velocity distribution to be biased toward low velocities because more objects are attributed distances too small than too large. This bias is stronger for the LAS sample but does not represent a major distortion of the distribution.

It is possible that there are subclasses of radio pulsars that have been severely biased against in pulsar searches, such as long lived millisecond pulsars (J.H. Taylor, this volume), which may rise to large distances from the galactic plane. Such a subclass is probably no more than about $10 \%$ of all pulsars, so any modification to the velocity distribution is minor (especially since this subclass is likely to be a low velocity component). Finally, although there are biases against precision measurement of high or low velocity objects inherent to the interferometry and scintillation techniques (as discussed above), these are at such extreme velocities that the derived distribution is essentially unaffected.

The velocity distribution is an important boundary condition for the stellar evolution of neutron star progenitors. Accounting for it with published models of evolution of single stars and of stars binary systems goes hand in hand with an accounting for the fraction of binary radio pulsars $(\sim 1.6 \%)$. This is discussed further below and in Dewey and Cordes (this volume).

\section{CORRELATIONS OF $V_{\perp}$ WITH OTHER PULSAR PARAMETERS}

Table 2 shows correlation coefficients (along with $99 \%$ confidence intervals) of transverse speed with other pulsar parameters, including $P \dot{P}$; the 'initial' value $P \dot{P}_{i}$ calculated assuming decay of the magnetic field on a time scale $\tau_{B}=9 M y r$; the spindown time $\tau_{S} \equiv P / 2 P$; the chronological age $t \equiv 0.5 \tau_{B} \ln \left(1+2 \tau_{S} / \tau_{B}\right)$; distance $\mathrm{D}$; distance from the galactic plane $|z|=D \sin |b|$; the $\mathrm{z}$ velocity estimate $V_{z} \equiv|z| / t$; and radio 'luminosity' $L \equiv S_{400} D^{2}$, where $S_{400}$ is the $400 \mathrm{MHz}$ flux density.

Correlation coefficients were calculated between logarithms of the various 
TABLE 2 CORRELATONS OF $V_{\perp}$ WITH OTHER PARAMETERS

\begin{tabular}{ccc}
\hline Parameter & $\begin{array}{c}\text { LAS Sample } \\
(26 \text { objects })\end{array}$ & $\begin{array}{c}\text { Scintillation Sample } \\
(59 \text { objects })\end{array}$ \\
\hline
\end{tabular}

$\begin{array}{ccc}\mathrm{PP} & 65(+22,-42) \% & 53(+20,-29) \% \\ (\mathrm{P} \dot{\mathrm{P}})_{\mathrm{i}} & 61(+24,-44) & 33(+27,-33) \\ \tau_{\mathrm{S}} & -39(+51,-35) & -41(+32,-24) \\ \mathrm{t} & -29(+52,-39) & -36(+33,-26) \\ |z| & 59(+25,-45) & 14(+31,-34) \\ \mathrm{D} & 54(+27,-47) & 27(+28,-34) \\ \mathrm{V}_{\mathrm{z}} & 61(+24,-44) & 43(+24,-31) \\ \mathrm{L} & 41(+34,-51) & 23(+29,-33)\end{array}$

quantities and are given for both the LAS sample of 26 interferometry derived velocities and for 59 of the scintillation velocities. The 59 object sample results from the removal of 'outlier' points which are objects with poorly measured velocities (probably due to leverage effects described above). Spearman rank correlations were also calculated and agree with those shown in Table 2, indicating that the coefficients are representative of all objects in the samples and are not dominated by just a few objects. Details of the correlation analysis may be found in Cordes (1986).

The velocity is correlated at significant levels with many of these parameters. Correlations with quantities involving distance are larger for the LAS sample than for the scintillation sample. This may be due to the fact that distances are in error and interferometer derived velocities are more sensitive to the distance. The correlation of velocity with $V_{z}$, however, is consistent with most pulsars being born in the galactic plane. A perfect correlation between transverse and $\mathrm{z}$ velocities is not expected because of geometrical decorrelation and from the fact that there exist runaway progenitors of neutron stars. The level of correlation is too large to be explained solely by errors in the distance scale. This may be checked by the correlation of $V_{\perp} / D$ with $\sin |b|$, which is at the level of $42 \%$ for the two samples. Since $\sin |b|$ is independent of distance, this level of correlation cannot be explained by errors in the distance scale.

For quantities in Table 2 involving $\dot{P}$, the velocity is most correlated with $P \dot{P}$, which is conventionally associated with the torque, and for magnetic dipole models of the magnetosphere, $P \dot{P} \propto$ (magnetic moment) $^{2}$. This correlation was first pointed out by Helfand and Tademaru (1977) and verified by Anderson and Lyne (1983). Figure 6 shows the correlation coefficient between $\log V_{\perp}$ and $\log P^{\alpha} \dot{P}$ plotted against $\alpha$. Although $\alpha$ is not well constrained, a value of 1 is certainly consistent with both velocity samples. The surprising result is that the velocity has anything to do with $P$ and $\dot{P}$.

Since the correlation with $P \dot{P}$ is potentially very interesting, I have investigated it for various subsamples of pulsars (Cordes 1986). It appears significant for all such subsamples and does not appear to arise from any peculiarity in those 


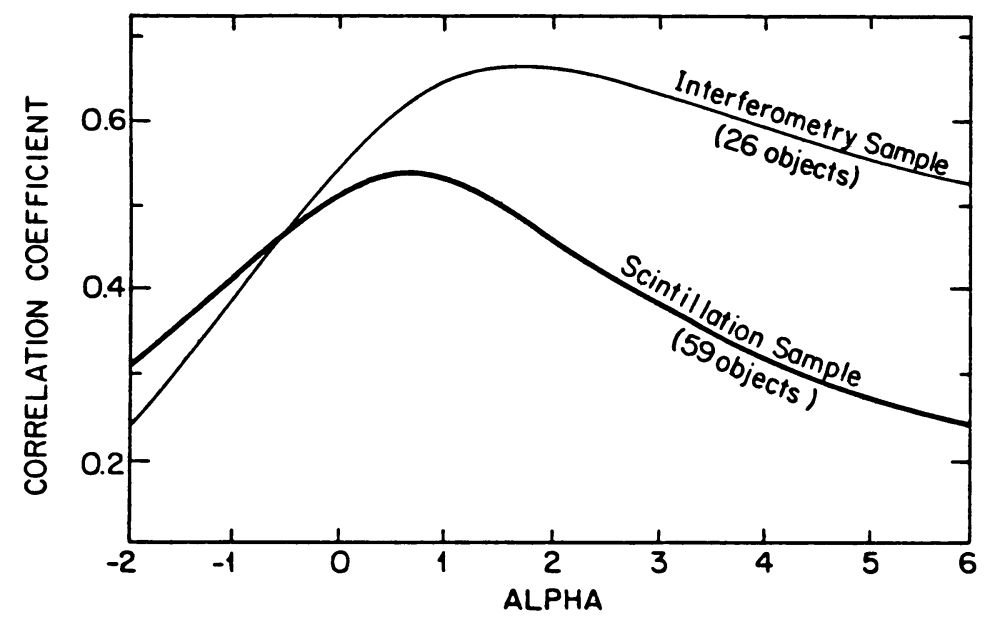

Figure 6 Correlation coefficient between logarithms of $V_{\perp}$ and $P^{\alpha} \dot{P}$ as a function of $\alpha$.

objects that are near the Earth or to be due to any correlation of radio luminosity with $P \dot{P}$ combined with selection effects in pulsar searches. Cheng (1985) has attempted to account for the correlation with spindown models involving (velocity dependent) accretion of grains into the pulsar magnetosphere. Other aspects of the pulsar population are inconsistent with this model, however, such as a predicted relation of $\dot{P}$ with $|z|$. Thus, I conclude that the space velocity of a radio pulsar is generally correlated with $P \dot{P}$, possibly signifying a correlation of space velocity with magnetic moment of the neutron star itself.

\section{DISCUSSION}

The distribution of transverse space velocities is quite broad and it is clear that pulsars as a class are high velocity objects. Moreover, there are a number of significant correlations between transverse velocity and other parameters, especially the quantity $P \dot{P}$.

It has long been suspected that pulsar velocities are related to their genesis in (primarily) binary systems, most of which are disrupted by mass loss associated with supernova explosions (Gunn and Ostriker 1970). Only until now, however, has it become possible to test scenarios for neutron star formation against observational constraints, such as the velocity distribution. Given the large variety of evolutionary paths that may lead to the formation of radio pulsars, (e.g. van den Heuvel, these proceedings), it is likely that the velocity distribution is a mixture of distributions for different paths.

Dewey and Cordes (1986) have undertaken a Monte Carlo simulation of neutron star progenitors that uses the known statistics of massive stars (distributions in masses, binary membership, orbital period) and conventional ideas about mass exchange and mass loss to predict the velocity distribution and binary membership of radio pulsars. Selection effects have also been studied using simulations and the 
results show that the velocity distribution is largely unaffected.

Preliminary results show that if supernova explosions are symmetric, then (1) the resultant velocity distribution contains far too many low velocity objects and (2) there should be a much larger fraction of radio pulsars in binary systems than is currently known. To 'fix' the velocity distribution (while keeping the assumption of symmetric explosions), there would have to be many more compact binaries $\left(P_{\text {orb }}<1\right.$ day) than are implied in the work of Abt (1983). A simpler route involves relaxation of the assumption of perfectly symmetric explosions. It is found that an extra kick velocity $\sim 90 \mathrm{~km} \mathrm{~s}^{-1}$ is sufficient to account for both the velocity distribution and the number of binary radio pulsars (Dewey and Cordes, this volume).

I thank R. Dewey for many useful discussions. I also thank D. Backer, A. Blaauw, A. Lyne, R. Narayan, E. Tademaru, and A. Wolszczan for informative discussions. This research was supported by NSF grant AST-8311844 to Cornell University, by the Alfred P. Sloan Foundation, and by the National Astronomy and Ionosphere Center, which operates Arecibo Observatory under contract with the NSF.

\section{REFERENCES}

Abt, H.A. 1983, Ann. Rev. Ast. Ap., $21,343$.

Anderson, B. and Lyne, A.G. 1983, Nature, 303, 597.

Armstrong, J.W., Cordes, J.M., and Rickett, B.J. 1981, Nature, 291,561.

Blaauw, A. 1961, B.A.N., 15, 265.

Cheng, A. 1985, Ap.J., $299,917$.

Cordes, J.M., Weisberg, J.M., and Boriakoff, V. 1985, Ap.J., 288,221.

Cordes, J.M. 1984 in IAU Symposium 110 VLBI and Compact Radio Sources,

eds. R.Fanti, K.Kellerman, and G. Setti (Dordrecht: Reidel), p. 303.

Cordes, J.M., 1986, Ap.J., in press (1 Dec).

Gunn, J.E. and Ostriker, J.P. 1970, Ap.J., 160, 979.

Harrison, E.R. and Tademaru, E. 1975, Ap.J., $201,447$.

Helfand, D.J. and Tademaru, E. 1977, Ap.J., $216,842$.

Lyne, A.G. 1984 Nature, 310, 300.

Lyne, A.G., Anderson, B., and Salter, M.J. 1982, M.N.R.A.S., 201, 503.

Lyne, A.G., Manchester, R.N., and Taylor, J.H. 1985, M.N.R.A.S., 213, 613.

Radhakrishnan, V. 1984 in Millisecond Pulsars, eds. S.P. Reynolds and

D.R. Stinebring (NRAO: Green Bank), 130.

Rickett, B.J., Coles, W.A., and Bourgois, G. 1984, Ast. Ap., 134, 390.

Simonetti, J., Cordes, J.M., and Spangler, S.R. 1984, Ap.J., 284, 126.

Zwicky, F. 1957, Morphological Astronomy (Berlin: Springer-Verlag), 258.

\section{DISCUSSION}

B. Basinska-Lewin: Ionospheric scintillations can cause significant variations in phase and intensity of radio signals at VHF frequencies. Is it possible that motion of ionospheric plasma irregularities could bias your results towards low velocities and contribute to the relatively larger number of pulsars with perpendicular velocities in your sample? 
J. Cordes: Unlike interstellar scintillations, ionospheric scintillations are broadband and their influence on the observed fluctuations in time and frequency can be calibrated out. Moreover, even if there were residual leakage of ionospheric scintillations into the net results, they would tend to shorten the derived fluctuation time scale. This would imply a bias to large velocities. In any case, this bias is negligible.

R. Becker: Does the correlation between $u$ and $v_{z}$ indicate anything other than the fact that both depend on the assumed distance?

J. Cordes: Yes. The correlation is too large to be due to the distance dependence.

D. Backer: If $P \dot{P}$ is perfectly correlated with the total pulsar space velocity, what correlation coefficient is expected between $P \dot{P}$ and the perpendicular velocity? If this is so close to the observed correlation, can we then infer radial velocities from $P \dot{P}$ and $V_{\perp}$ ?

J. Cordes: As it turns out, the correlation of perpendicular velocity with $\mathrm{P} \dot{P}$ would be identical with the correlation between total velocity and $\mathrm{PP}$. This is because, although geometrical effects lessen the average cross product of $V_{\perp}$ and $\mathrm{P} \dot{\mathrm{P}}$, they also lessen the standard deviation of $\mathrm{V}_{\perp}$ with respect to total velocity. Thus the normalized correlation coefficient is unchanged.

E. van den Heuvel: There is something which I do not understand. From Lyne's talk I understand that ( $P \dot{P})$ decreased in the course of time. If the correlation between ( $P \dot{P})$ and $V$ would be causal, this would mean that the velocity decreases in time. This seems rather odd to me.

J. Cordes: Your argument is amusing but not physical. Decay of $P P$ decreases only the correlation coefficient between $V$ and $P \dot{P}$ (assuming that $V_{1}$ is correlated with $\mathrm{PP}$ at birth). Different pulsars of course have different ages and perhaps different decay times. Thus one should expect the correlation between $V_{1}$ and $P \dot{P}$ to be imperfect, especially since there are multiple physical contributions to $\mathrm{V}$. I should add that the correlation coefficient $V_{1}$ and initial $P \dot{P}$ (assuming a universal decay time constant) is somewhat smaller than between $V$ and current PP. I would argue that we do not understand torque decay well enough to extrapolate backwards in time.

E. van den Heuvel: One of my students, G. Stollman (1986), did a Monte Carlo simulation of the expected observable pulsar population, taking selection effects into account and found this leads to an expected observational correlation between the magnetic field strength $B$ and space velocity. This is a purely spurious correlation, produced by selection effects, and of the same order of magnitude as the observed correlation.

J. Cordes: This is contrary to the results of simulations reported by Dewey and myself (this volume). As I recall from the paper (Stollman and van den Heuvel 1986) the level of correlation obtained with your treatment of selection effects is much smaller than the observed level of correlation. I think that saying the correlation is spurious is misrepresentative of your own 
simulation and certainly misrepresentative of the observational results.

S. Kulkarai: Does your model reproduce the observed fraction of binary pulsars? Does it reproduce the velocity distribution of runaway $O B$ stars?

J. Cordes: The simulation involving symmetric supernova explosions produces far too many binary radio pulsars and $B$ star velocities that are too smal1. Perhaps binary radio pulsars should be searched for, but other evidence (the pulsar velocity distribution) strongly suggests that other effects (e.g., asymmetric explosions) in addition to disrupted orbital motion are involved and that such binaries do not exist.

D. Eichler: The electromagnetic rocket mechanism (Harrison and Tademaru) would explain the $\mathrm{PP}-\mathrm{V}_{\perp}$ correlation in a straightforward manner, wouldn't it?

J. Cordes: Perhaps. But remember the net translational velocity produced by this mechanism is independent of the magnetic moment (large moments produce a bigger acceleration, but for a shorter time). Thus one would need something like a relationship between magnetic moment and its offset from the neutron star axis to explain the correlation.

R. Manchester: The rocket mechanism for accelerating pulsars is unlikely to be effective as the force depends on the fifth power of the pulsar rotation frequency and there is now good evidence that pulsars are generally born with periods which are not very short.

J. Cordes: The question is what you mean by generally. Are you certain that no strong magnetic field pulsars are born with millisecond periods? I think the period distribution is still uncertain for small periods because $I$ have not seen a complete treatment of selection effects. The evidence that pulsars are born with larger periods really is evidence that pulsars only spend small amounts of time at short periods. I contend that the jury is still out on the rocket mechanism.

A. Burrows: It might be pointed out that a velocity asymmetry is suggested in a scenario recently proposed by Stan Woosley and myse1f for PSR1913+16. It is encouraging to see indications of asymmetry from such independent work, though we, by no means, would conclude that all explosions are asymmetric in interesting ways.

J. Cordes: Yes. I recall from your preprint that your conclusion for $1913+16$ depends critically on the mapping of progenitor mass to remnant mass. Let me point out that Ira Wasserman and I conclude that if the explosion was asymmetric in the 1913+16 system so as to give a kick out of the pre-explosion orbital plane, then geodetic precession of the pulsar spin axis must be occuring. We are presently looking for this effect in polarization measurements from the Arecibo Observatory. 